\title{
S100A6 stimulates proliferation and migration of colorectal carcinoma cells through activation of the MAPK pathways
}

\author{
LIANG DUAN $^{1}$, RUI WU ${ }^{2}$, ZHENGYU ZOU ${ }^{1}$, HAIYAN WANG ${ }^{1}$, LIWEI YE ${ }^{1}$, HUAN LI $^{1}$, SHIMEI YUAN ${ }^{1}$, \\ XUERU LI ${ }^{1}$, HE ZHA ${ }^{1}$, HUI SUN ${ }^{1}$, YUNYUAN ZHANG ${ }^{1}$, XIAN CHEN $^{1}$ and LAN ZHOU ${ }^{1}$ \\ ${ }^{1}$ Key Laboratory of Diagnostic Medicine Designated by the Chinese Ministry of Education, \\ Chongqing Medical University; ${ }^{2}$ Department of Laboratory Medicine, The First Affiliated \\ Hospital of Chongqing Medical University, Chongqing 400016, P.R. China
}

Received October 25, 2013; Accepted December 2, 2013

DOI: 10.3892/ijo.2013.2231

\begin{abstract}
The S100A6 protein, a member of the S100 protein family, is overexpressed in many tumors including colorectal carcinoma (CRC). Although recent studies showed that the elevated expression of S100A6 was associated with the stage and lymphatic permeation of CRC, little is known about whether and how S100A6 contributes to CRC development. Here we investigated the S100A6 expression in CRC tissues and cell lines, and explored the molecular mechanisms underlying the role of S100A6 in CRC development by examining cell proliferation and migration in vitro, and tumorigenicity in nude mice. The results show that S100A6 expression was markedly increased in CRC tissues and cell lines compared to normal colon tissues and a normal colon mucosal epithelial cell line, respectively. Recombinant adenovirus-mediated overexpression of S100A6 or treatment with recombinant S100A6 protein in HCT116, a CRC cell line with relative low S100A6 expression, resulted in enhanced cell proliferation and migration, and the mitogen-activated protein kinase (MAPK) activation in vitro, and tumor growth in vivo. Conversely, RNAi-mediated knockdown of S100A6 in LoVo, a CRC cell line with relative high S100A6 expression, resulted in reduced cell proliferation, migration and MAPK activity. S100A6induced proliferation was partially attenuated by an ERK inhibitor while migration was suppressed by a p38 inhibitor. Taken together, our results suggest that the cellular effects of S100A6 are mediated by the ERK and p38 MAPK pathways, and modulation of these pathways may be employed for CRC prevention and therapy.
\end{abstract}

Correspondence to: Dr Lan Zhou, Key Laboratory of Diagnostic Medicine Designated by the Chinese Ministry of Education, Chongqing Medical University, 1 Yixueyuan Road, Yuzhong District, Chongqing 400016, P.R. China

E-mail: zhoulan0111@foxmail.com

Key words: S100A6, proliferation, migration, colorectal carcinoma

\section{Introduction}

Colorectal carcinoma (CRC) is one of the most common malignant tumors and the third leading cause of cancer-related death worldwide (1). Although novel molecule-based therapies are widely used in treating $\mathrm{CRC}$, the high recurrence and poor survival remain as risk factors to many CRC patients (2). Thus, it is necessary to elucidate the molecular mechanisms in the pathogenesis and progression of CRC and to develop novel therapeutic strategies to conquer this malignancy.

S100A6, a member of the S100 calcium-binding family proteins, was originally purified from Ehrlich ascites tumor cells and subsequently detected in various cell types such as fibroblasts, epithelial cells, neurons, smooth muscle cells and lymphocytes $(3,4)$. For its biological functions, knockdown of S100A6 strongly inhibited proliferation of fibroblasts, osteoblasts and pancreatic carcinoma cells (5-7). On the other hand, overexpression of S100A6 results in enhanced osteoblast proliferation (6). Furthermore, S100A6 impacts adhesion and motile properties of pancreatic cancer cells and osteosarcoma cells $(8,9)$. Additionally, several studies reported that S100A6 is detected in the culture medium or microenvironment and exert its extracellular roles through binding to the transmembrane receptor for advanced glycation end products (RAGE) and activating the downstream signaling pathways $(4,10,11)$. While the human S100A6 gene is located in chromosome 1q21, an unstable region that is rearranged in tumors, its abnormal expression has been found in many tumors (12-14). S100A6 is upregulated and correlated with Dukes' tumor stage or lymphatic permeation in CRC $(15,16)$. S100A6 is transcriptionally regulated by $\beta$-catenin, a key effector of Wnt/ $\beta$-catenin signaling pathway that is abnormally activated in CRC (17). However, the effect of S100A6 on CRC and the underlying molecular mechanisms are still elusive.

The mitogen-activated protein kinase (MAPK) signaling pathways play critical roles in pathogenesis, progression, and oncogenic behavior of CRC (18). Recent studies show that some members of the S100 family exert their biological effects involving activation of MAPK signaling pathways. For example, S100P enhances tumor cell proliferation and regulates cytoskeletal dynamics involving MAPK activation (19). S100A8 and S100A9 promote breast cancer cell growth 
and gastric cancer cell migration via the MAPK signaling pathways (20-22). S100A12 participates in the development of osteoarthritis involving MAPK signaling (23). S100A14 stimulates esophageal squamous cell carcinoma cell proliferation via MAPK activation (24). However, it is still unclear whether S100A6 regulates the MAPK signaling pathways for CRC progression.

In the present study, we investigated the effects of S100A6 on CRC cell proliferation and migration and the underlying molecular mechanisms. The results suggest that S100A6 promotes CRC cell proliferation and migration through activation of the ERK and p38 MAPKs, which could be targeted for CRC prevention and therapy.

\section{Materials and methods}

Tissues. Fresh colorectal cancer tissues and matching distal normal tissues were collected from 10 patients who had undergone colorectal resection at the First Affiliated Hospital of the Chongqing Medical University. The patients received no chemotherapy, hormonal therapy or radiotherapy before surgery, and a written informed consent was received from all participants. This study was approved by the Ethics Committee of Chongqing Medical University (protocol no. 2012-19).

The freash samples from these patients were stored at $-80^{\circ} \mathrm{C}$ and were used for western blot analysis. The specimens were fixed in $10 \%$ buffered-formalin, embedded in paraffin blocks and were serially sectioned for hematoxylin-eosin (H-E) and immunohistochemical staining. These sections were viewed twice by two pathologists in a blinded manner to verify the diagnosis, histological differentiation, and pathological stage.

Cell lines. Human colorectal carcinoma cell lines HCT116, SW480 and LoVo were purchased from ATCC (American Type Culture Collection, Manassas, VA, USA). Human normal colon mucosal epithelial cell line NCM460 and human embryonic kidney cell line 293 (HEK293) were purchased from China Center for Type Culture Collection (CCTCC). HCT116, LoVo and HEK293 cells were maintained in Dulbecco's modified Eagle's medium (DMEM) with 10\% FBS (Hyclone, USA), and SW480 and NCM460 cells were cultured in Roswell Park Memorial Institute (RPMI)-1640 with 10\% FBS (Gibco, USA). Cell culture was maintained at $37^{\circ} \mathrm{C}$ in a humid atmosphere containing $5 \% \mathrm{CO}_{2}$.

Reagents and antibodies. The primary antibodies used in this study were: mouse anti-hS100A6 monoclonal antibody (cat. no. 3950; Santa Cruz Biotechnology, Santa Cruz, CA, USA), rabbit anti-Ki67 polyclonal antibody (cat. no. YT2467; ImmunoWay, Newark, DE, USA), rabbit anti-JNK monoclonal antibody (cat. no. 9253; Cell Signaling Technology, Danvers, MA, USA), rabbit anti-phosphor-JNK monoclonal antibody (cat no. 4668; Cell Signaling Technology), rabbit anti-p38 monoclonal antibody (cat. no. 9212; Cell Signaling Technology), rabbit anti-phosphor-p38 monoclonal antibody (cat. no. 4511; Cell Signaling Technology), rabbit anti-ERK1/2 monoclonal antibody (cat. no. 4695; Cell Signaling Technology), rabbit anti-phosphor-ERK1/2 monoclonal antibody (cat. no. 3510; Cell Signaling Technology) and mouse anti- $\beta$-actin mono- clonal antibody (cat. no. 47778; Santa Cruz Biotechnology). Specific inhibitors of p38 (SB203580) and ERK1/2 (PD98059) were obtained from Santa Cruz Biotechnology, Inc. and were used as per the manufacturer's instructions.

Immunohistochemistry (IHC). The expression of S100A6 and $\mathrm{Ki} 67$ in tissues was examined by IHC. The sections from the formalin fixed, paraffin-embedded tissues were deparaffinized and dehydrated. Then the sections were boiled for $10 \mathrm{~min}$ in $0.01 \mathrm{M}$ citrate buffer and incubated with $0.3 \%$ hydrogen peroxide $\left(\mathrm{H}_{2} \mathrm{O}_{2}\right)$ in methanol for 15 min to block endogenous peroxidase. The sections were then incubated with the anti-S100A6 monoclonal antibody (1:300 dilution) and rabbit anti-Ki67 polyclonal antibody (1:300 dilution) overnight at $4^{\circ} \mathrm{C}$, following incubation with secondary antibody tagged with the peroxidase enzyme (cat. no. SP-9000; Zhongshan Golden Bridge, Beijing, China) for $30 \mathrm{~min}$ at room temperature and were visualized with $0.05 \%$ 3,3-diaminobenzidine tetrachloride (DAB) till the desired brown reaction product was obtained. The sections were finally counterstained with hematoxylin. Control sections were performed using phosphate buffer solution (PBS) without a primary antibody. All slides were observed under a Nikon E400 Light microscope and representative images were taken. The immunohistochemical labeling was assessed by two pathologists. The results of immunohistochemistry staining were analyzed by a simplified score ranging from 0 to 4 for positive proportion as described before (25). The proportion of staining of tumor cells was classified into grade $1(<10 \%$ of positive cells), grade 2 (10-25\% of positive cells), grade 3 (26-75\% of positive cells) and grade 4 ( $>75 \%$ of positive cells). In addition, the staining intensity was also scored in four grades (no staining, 0; weak, 1; moderate, 2; strong, 3). The staining index was obtained by multiplication of proportion and intensity ranging from 0 to 12 , and their expression with staining index of $\geq 2$ was regards as positive.

Immunofluoresence staining. The cells were plated and cultured onto cleaned-up cover slips, and were washed with PBS and fixed in $4 \%$ paraformaldehyde, then permeabilized with $0.2 \%$ Triton X-100. Cover slips were rinsed and incubated with blocking serum (goat serum) for $15 \mathrm{~min}$ at $37^{\circ} \mathrm{C}$ and then incubated with primary rabbit anti-hS100A6 monoclonal antibody (1:50 dilution) overnight at $4^{\circ} \mathrm{C}$. After three washes with PBS, the cells were stained with the corresponding FITCconjugated secondary antibody (1:100 dilution, ZF-0312; Zhongshan Golden Bridge, Beijing, China). To visualize nuclei, cells were stained with $10 \mu \mathrm{g} / \mathrm{ml}$ DAPI. The fluorescent images were then observed and analyzed using a laser scanning confocal microscope.

Preparation of S100A6 protein and amplification of recombinant adenoviruses. The recombinant human S100A6 (rhS100A6) protein used in this work has been described previously (26). Briefly, the plasmids pGST-Moluc-HRV3ChS100A6 and pGST-Moluc-HRV3C were transformed into $E$. coli (BL21) per the instructions of calcium chloride transformation. Isopropylthio- $\beta$-D-galactoside was used to induce the expression of GST-HRV3C-hS100A6 and pGSTMoluc-HRV3C proteins. Then the bacteria were collected 
and sonicated on ice, and spun at $4^{\circ} \mathrm{C}$. The supernatant was incubated with glutathione-Sepharose 4B beads, and GST-HRV3C-hS100A6 and GST-HRV3C on the beads were eluted by elution buffer with reduced glutathione on ice. GST-HRV3C-hS100A6 was digested by GST-HRV3C overnight in $4^{\circ} \mathrm{C}$, and then the GST tag and GST-HRV3C were removed by glutathione-Sepharose $4 \mathrm{~B}$ beads. Finally, the rhS100A6 protein was filtered and stored at $-80^{\circ} \mathrm{C}$.

The recombinant adenoviruses carrying human S100A6 gene (AdS100A6) and S100A6-siRNA gene (AdsiS100A6) and their control (AdGFP or AdRFP) were kindly presented by T.C. He (Medical Center, The University of Chicago), and all these adenoviruses were amplified in HEK293 cells before use $(9,27)$.

Coomassie brilliant blue staining. The recombinant protein S100A6 was subjected to polyacrylamide gel electrophoresis. Following electrophoresis, the gel was placed in a colloidal coomassie staining solution and incubated for $6 \mathrm{~h}$ to overnight. Then distilled water was used to de-stain the gel until the background was transparent. All steps were done on a rotary shaker with slight mixing.

Cell proliferation assay. Cell proliferation was measured using MTT (3-(4,5-dimethylthiazol-2-yl)-2,5-diphenyltrazolium bromide) assay. Cells $\left(3 \times 10^{3}\right)$ were seeded into each well of 96-well culture plates, grown for $24 \mathrm{~h}$, then treated with rhS100A6 protein, AdS100A6, AdGFP (control for AdS100A6), AdsiS100A6 and AdRFP (control for AdsiS100A6) in DMEM containing 1\% FBS for 24, 48 ,72, and $96 \mathrm{~h}$. After the indicated hours of incubation, the MTT reagent (Promega, Madison, WI, USA) was added (20 $\mu \mathrm{l} /$ well) and incubated for $4 \mathrm{~h}$ at $37^{\circ} \mathrm{C}, 100 \mathrm{ml}$ dimethyl sulfoxide was added to dissolve the formazan product for $10 \mathrm{~min}$ at room temperature. The absorbance was measured daily for the following five days at $492 \mathrm{~nm}$ using a microplate reader. Each condition was done in quintuplicate, and the experiment was repeated thrice.

Tumorigenicity assays in nude mice. The in vivo experiments were performed as previously described (22). All the experimental procedures were conducted in accordance with the guidelines established by the University Animal Care and Use Committee for Laboratory Animal Research. Briefly, the 6-8-week old female nude mice were randomly divided into 4 groups ( $n=4 /$ group). Untreated, AdGFP-treated, AdS100A6treated and rhS100A6 (protein, $20 \mu \mathrm{g} / \mathrm{ml}$ )-treated HCT116 cells ( $2 \times 10^{7} /$ each nude mouse) were suspended in $200 \mu 1 \mathrm{PBS}$, and then were injected subcutaneously into the posterior flank position of nude mice. Subcutaneous tumor growth was recorded every 5 days with vernier calipers. Tumor volume was calculated using the formula: $\pi / 6 \times\left(\mathrm{R} \max \times \mathrm{R} \min ^{2}\right)$, where $\mathrm{R}$ is the tumor diameter. The mice were sacrificed after 60 days, and the tumor tissues were collected, fixed in buffered formaldehyde, embedded in paraffin, and sectioned for further histological and immunohistochemical analysis.

Cell migration assay. Cell migration ability was analyzed by means of transwell migration assay and wound scratch assay. The transwell migration assay was performed as previously described (25). The cells were seeded in the upper chamber of non-type I-collagen-coated 24-well culture inserts. After $24 \mathrm{~h}$, the cells were dried for $5 \mathrm{~min}$, fixed with dehydrated alcohol, and stained with hematoxylin-eosin. The cells that invaded the collagen-coated-inserts were counted. Mean values for five randomly selected fields were obtained for each well. The experiments were performed and repeated thrice. Values are expressed as the mean \pm standard deviation.

For the wound scratch assay, log-phase cells were collected and seeded in 6-well plates and were treated with AdS100A6, AdsiS100A6, AdGFP and AdRFP. After treating for indicated time, a wound was created at the center of the culture using a pipette tip, and the cells were washed with serum-free medium, cultured with $1 \%$ FBS. Images were taken under a microscope immediately after the incision was made. The incision width of the different sites was measured, and the average woundclosure rate was calculated. The wound-closure rate was calculated as: ( $0 \mathrm{~h}$ incision width $-72 \mathrm{~h}$ incision width) $/ 0 \mathrm{~h}$ incision width $\mathrm{x} 100 \%$.

Western blot assay. The tissues and cells were collected and washed with ice-cold PBS, then lysed on ice in radio immunoprecipitation assay (RIPA) buffer The total lysate was centrifuged and the proteins in the supernatant were quantitated by BCA (bicinchoninic acid) assay and denatured by boiling and loaded onto a $10 \%$ sodium dodecyl sulphatepolyacrylamide gel electrophoresis (SDS-PAGE) and blotted onto the PVDF membranes. Then the membranes were blocked with $5 \%$ bovine serum albumin at room temperature for $2 \mathrm{~h}$ and incubated with anti-S100A6 monoclonal antibody $(1: 1,000$ dilution), or anti-phosphor-p38 monoclonal antibody $(1: 1,000$ dilution), or rabbit anti-p38 monoclonal antibody (1:1,000 dilution), or rabbit anti-phosphor-ERK1/2 monoclonal antibody (1:1,000 dilution), or rabbit anti-ERK1/2 monoclonal antibody (1:1,000 dilution), rabbit anti-phosphor-JNK antibody $(1: 1,000$ dilution), or rabbit anti-JNK monoclonal antibody (1:1,000 dilution), or mouse anti- $\beta$-actin monoclonal antibody $(1: 1,000$ dilution). The secondary antibodies included goat anti-rabbit IgG serum (1:5,000 dilution) or goat anti-mouse IgG serum (1:5,000 dilution). The proteins of interest were detected using the SuperSignal West Pico Chemiluminescent Substrate kit. The results were recorded using the Bio-Rad Electrophoresis Documentation (Gel Doc 1000) and Quantity One version 4.5.0 software (Bio-Rad, Hercules, CA, USA).

Statistical analysis. All values in the text and figures are presented as the mean \pm standard deviation (SD). The differences were analyzed using one-way ANOVA followed by the Student-Newman-Keuls test, and all statistical analyses were performed using GraphPad Prism software (GraphPad Software, La Jolla, CA, USA). Statistical differences are presented at probability levels of $\mathrm{p}<0.05, \mathrm{p}<0.01$ and $\mathrm{p}<0.001$.

\section{Results}

Elevated expression of S100A6 in CRC tissues and cell lines. We examined the expression and distribution of S100A6 in human CRC tissues by IHC. While the S100A6 signal was present in a small number of interstitial cells of the normal tissues, which was much more intense in CRC tissues. The 


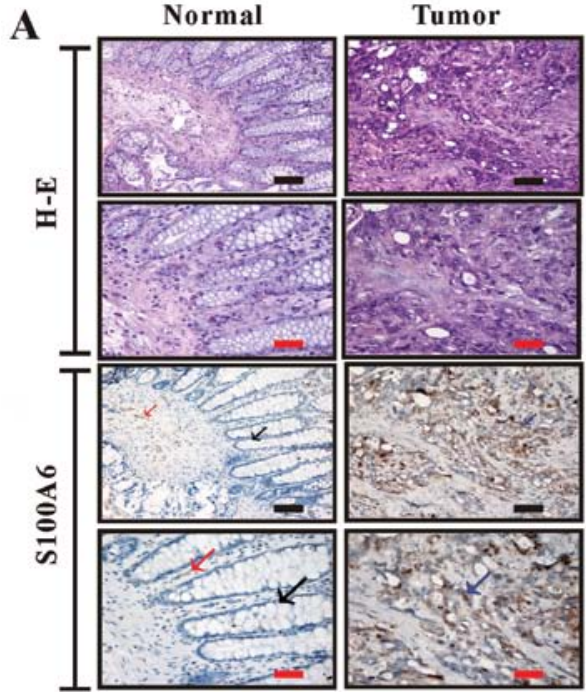

C

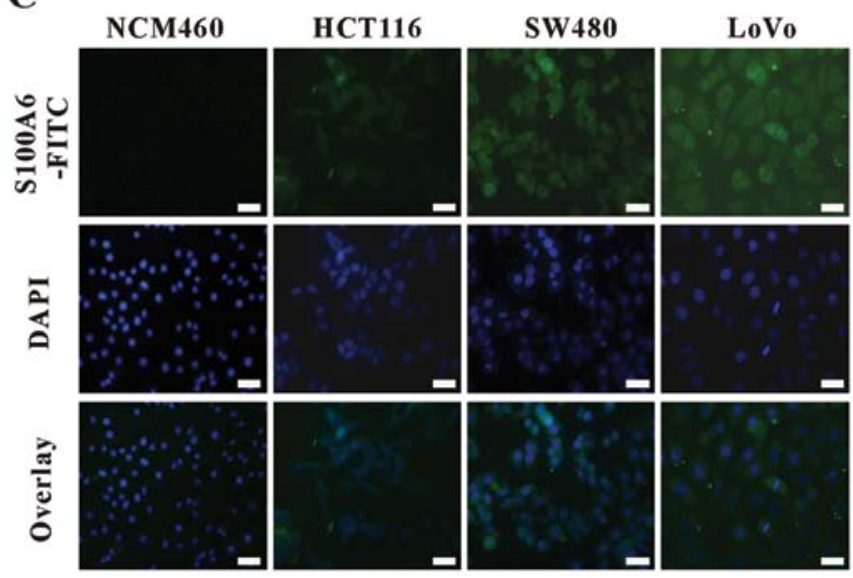

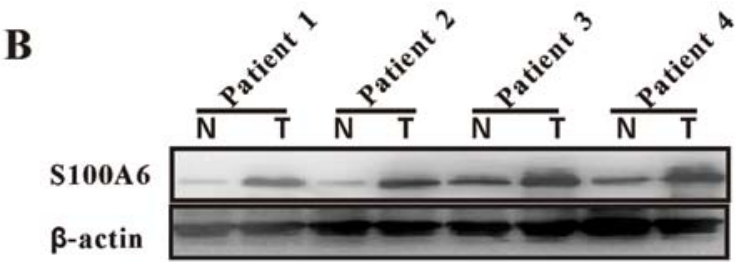

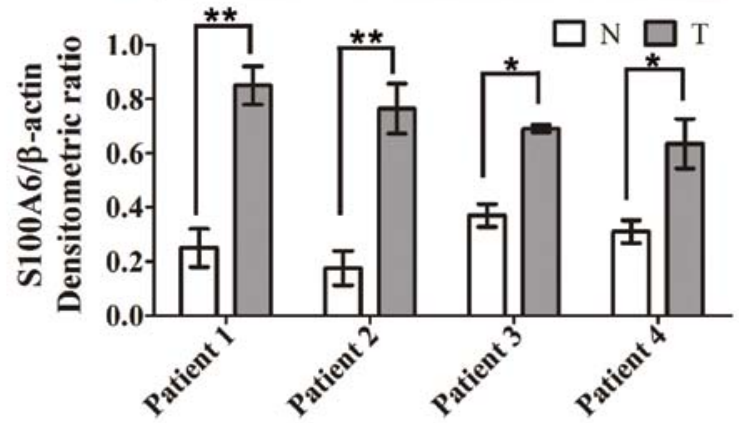

D
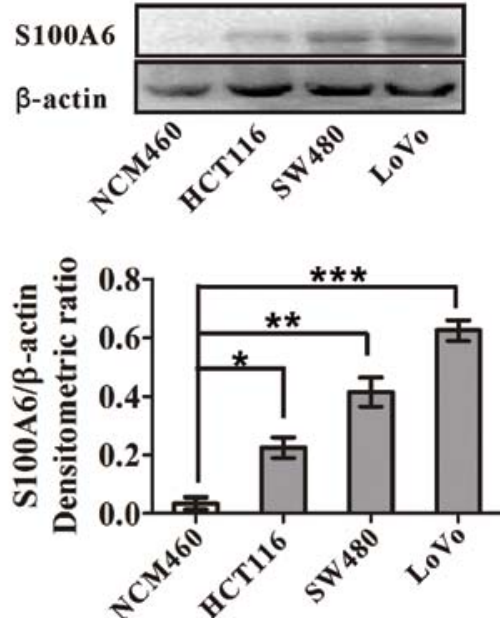

Figure 1. S100A6 is highly expressed in human colorectal carcinoma tissues and cell lines. (A) Immunohistochemistry for S100A6 in normal colon and CRC tumor tissues. Black arrow, normal glandular epithelial cells; red arrow, S100A6-expressing interstitial cells (brown); blue arrow, S100A6-expressing cancer cells (brown). Black scale bars, $100 \mu \mathrm{m}$; red scale bars, $200 \mu \mathrm{m}$. H-E, hematoxylin and eosin staining. (B) Western blot analysis for the S100A6 expression in colorectal carcinoma and matching distal normal tissues from four randomly selected patients. $\beta$-actin was used as an input control. The S100A6/ $\beta$-actin densitometric ratios are also shown. T, tumor tissues; $N$, matching distal normal tissues. " $p<0.05$ and ${ }^{* *} \mathrm{p}<0.01, \mathrm{~T}$ vs. N. (C) Immunofluorescence staining analyses for the expression of S100A6 in CRC cell lines (HCT116, SW480 and LoVo) and normal colon mucosal epithelial cell line (NCM460). The cells were stained by the antibody against S100A6, and then FITC-labeled secondary antibody was applied (green fluorescence). The nucleus was counterstained with DAPI (blue). The images were visualized under a laser scanning confocal microscope. Blank scale bars, $100 \mu \mathrm{m}$. (D) Western blot analyses for the S100A6 expression in colorectal carcinoma cell lines (HCT116, SW480 and LoVo) and normal colon mucosal epithelial cell line (NCM460). $\beta$-actin was used as an input control. The S100A6/ $\beta \beta$-actin densitometric ratios are also shown. " $<<0.05$, HCT116 vs. NCM460; ${ }^{* *}$ p $<0.01$, SW480 vs. NCM460; ${ }^{* * *}$ p $<0.001$, LoVo vs.NCM460.

S100A6 staining in tumors showed a diffuse distribution in the cytoplasm and nuclei of tumor cells and interstitial cells (Fig. 1A). The enhanced immunoreactivity for S100A6 was detected in all examined samples from 10 patients, which was confirmed by western blotting in randomly chosen four cases of the examined samples (Fig. 1B).

The expression of S100A6 in CRC cell lines was compared to that in a normal colon mucosal epithelial cell line (NCM460) by immunofluorescence staining and western blotting. S100A6 expression was detected in all the three CRC cell lines but not in NCM460 (Fig. 1C and D). Among the CRC cell lines, LoVo had the highest while HCT116 had the lowest S100A6 expression (Fig. 1C and D). Thus, we chose to knock down S100A6 in LoVo cells and enforce S100A6 expression in HCT116 cells for the following studies.
S100A6 promotes $C R C$ cell proliferation. We used adenovirus vectors expressing human S100A6 (AdS100A6) or recombinant human S100A6 (rhS100A6) protein to treat HCT116 cells that have relatively low S100A6 expression level, and used AdsiS100A6 expressing S100A6 siRNA to infect LoVo cells that have relatively high S100A6 expression level for S100A6 knockdown. S100A6 overexpression by AdS100A6 and knockdown by AdsiS100A6 were confirmed by western blotting (Fig. 2A and B). The prepared rhS100A6 protein was confirmed by coomassie blue staining and identified by specific anti-hS100A6 antibody by western blotting (Fig. 2C and D). Its purity was $>90 \%$ following quantifying by Quantity One Software after SDS-PAGE.

Cell proliferation was evaluated by MTT assay. AdS100A6-mediated overexpression of S100A6 in HCT116 cells stimulated cell proliferation at day 4 ( $p<0.01$, Fig. $2 \mathrm{E})$. 
A

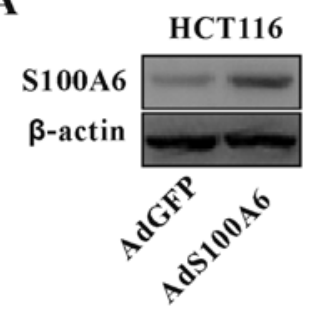

B

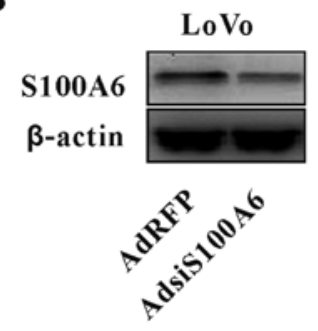

C

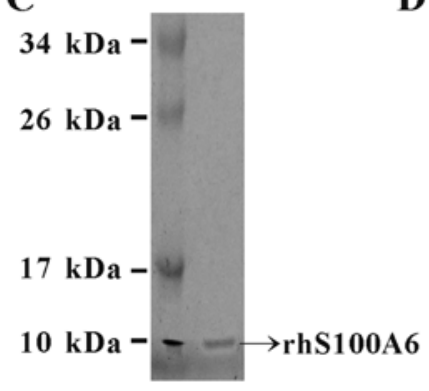

D

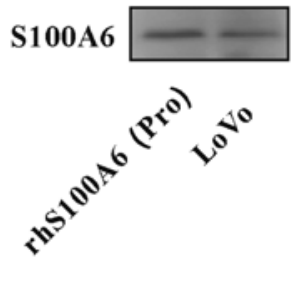

$\mathbf{E}$

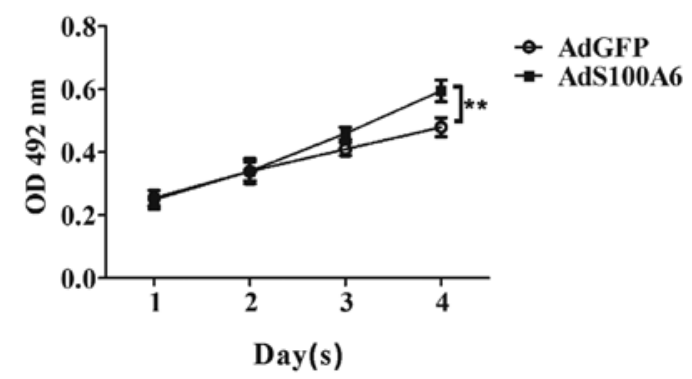

G
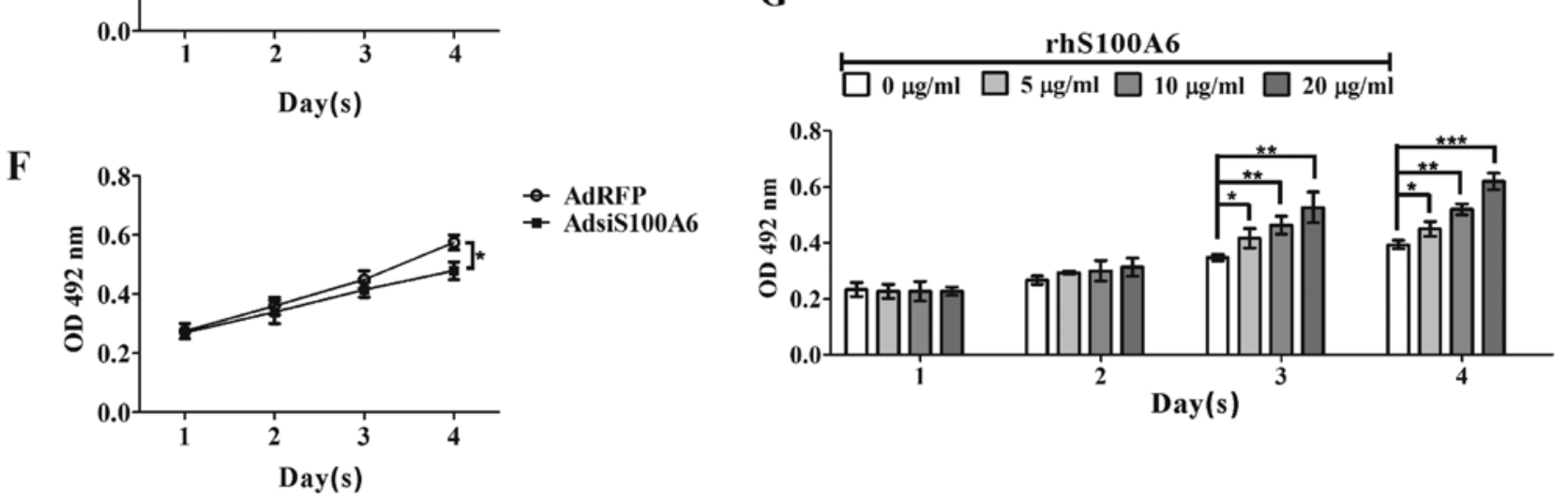

Figure 2. The effect of S100A6 on the proliferation of CRC cells. (A) HCT116 cells were infected with AdS100A6, and S100A6 expression was detected by western blotting. (B) LoVo cells were infected with AdsiS100A6, and S100A6 expression was detected by western blotting. (C) Identification of rhS100A6 protein by SDS-PAGE. kDa, kilodalton. (D) rhS100A6 was recognized by anti-S100A6 antibody through western blotting. Lane 1 , rhS100A6 protein; lane 2 , cell lysates (LoVo, control for S100A6). (E) HCT116 cells were treated with AdGFP or AdS100A6 continuously for four days, and cell proliferation was detected by the MTT assay. Results are expressed as the mean absorbance \pm SD of three independent experiments. ${ }^{* *}$ p $<0.01$, AdS100A6 vs. AdGFP control. (F) The proliferation of LoVo cells treated with AdRFP or AdsiS100A6 was detected using the MTT assay. *p<0.05, AdsiS100A6 vs. AdRFP control. (G) The proliferation of HCT116 cells treated with and without rhS100A6 at different concentrations was measured by the MTT assay. Results are expressed as the mean absorbance \pm SD of three independent experiments. ${ }^{*} \mathrm{p}<0.05,{ }^{* *} \mathrm{p}<0.01$ and ${ }^{* * *} \mathrm{p}<0.001$.

Conversely, AdsiS100A6-mediated knockdown of S100A6 in LoVo cells reduced cell proliferation at day $4(\mathrm{p}<0.05$, Fig. 2F). In addition, treatment with rhS100A6 protein also resulted in effective, enhanced proliferation of HCT116 cells concentration-dependently at days 3 and 4 (Fig. 2G).

S100A6 promotes tumorigenicity of CRC cells in vivo. We further investigated the effect of S100A6 on growth of CRC xenograft tumors in vivo. The 4 groups of HCT116 cells (untreated, AdGFP-treated, AdS100A6-treated and rhS100A6treated) were subcutaneously implanted into nude mice. Tumors became palpable from day 25 to 60 and continued to grow. While the tumors derived from the AdGFP-treated cells grew at a speed identical to that of tumors from the untreated control cells, the tumors derived from the AdS100A6-treated or rhS100A6-treated cells had a much more rapid growth rate (Fig. 3A), suggesting ectopic S100A6 expression promotes CRC tumor growth in nude mice. These results were further confirmed by IHC for Ki67 expression in tumor sections from different groups, showing that the immunoreactivity for Ki67 in group of AdS100A6 or rhS100A6 was much more intense than that in the AdGFP (control for AdS100A6) and untreated groups (control for rhS100A6) (Fig. 3B). Histological examination by $\mathrm{H}-\mathrm{E}$ staining showed that the tumor cells in all the groups were obviously heterogeneous, with large nuclei, high nucleus/cytoplasm ratios, irregular nuclear shapes, and variable nuclear sizes (Fig. 3B).

S100A6 promotes CRC cell migration. Cell migration plays a crucial role in the process of tumor invasion and metastasis. Wound healing assay was used to detect migration induced by overexpression of S100A6 in HCT116 and knockdown of S100A6 in LoVo cells. After overexpression of S100A6 by infecting HCT116 cells with AdS100A6 for $72 \mathrm{~h}$, wound closure rate was increased by $53.1 \%$ compared with that of the AdGFP group ( $p<0.05$, Fig. 4A). In contrast, knockdown of 
A

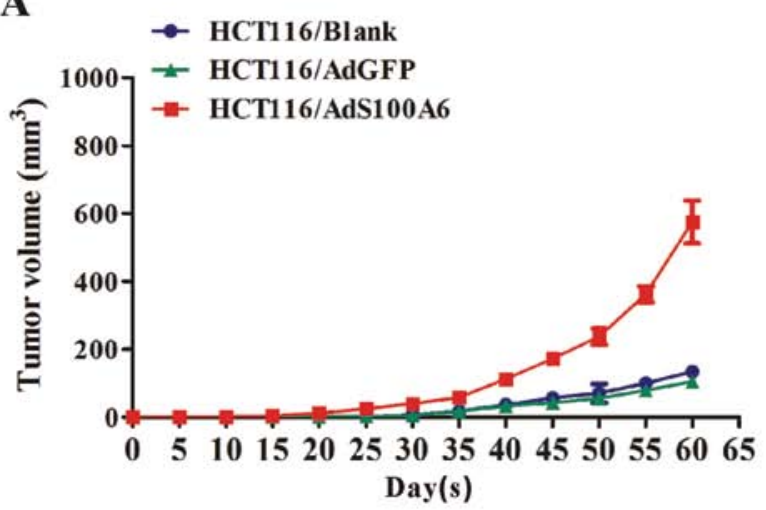

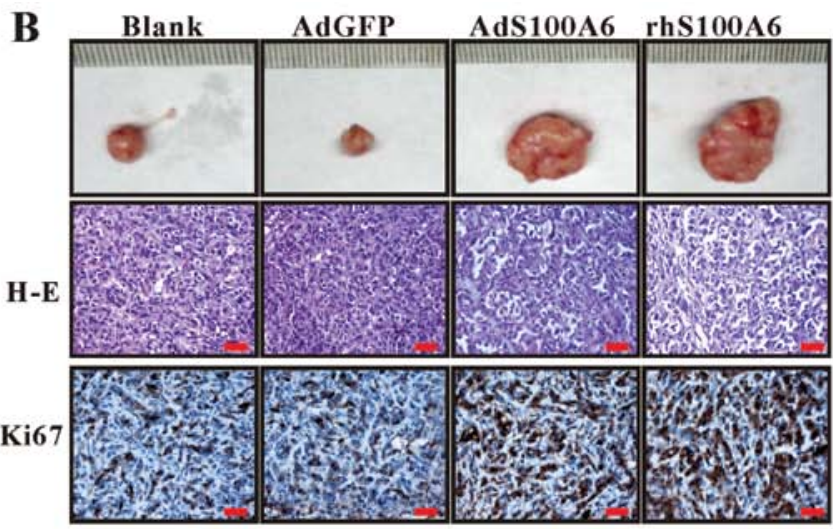

Figure 3. The effect of S100A6 on the growth of CRC in vivo. (A) Tumor growth curves of all groups (n=4/group). Tumor volume was measured every 5 days. (B) Iamges of representative excised tumor tissues along with Ki67 expression by immunohistochemistry analysis from blank (untreated), AdGFP-treated and AdS100A6-treated and rhS100A6-treated groups. The tumor tissues were confirmed by H\&E staining.

A
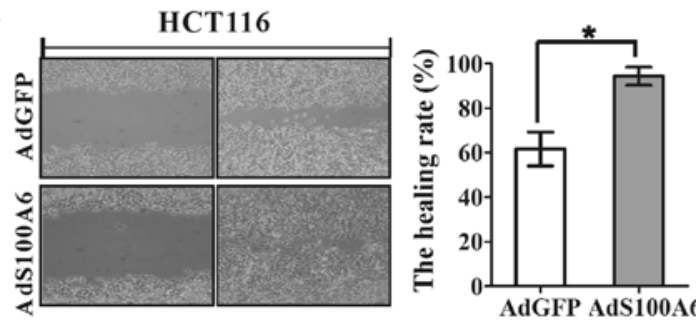

C
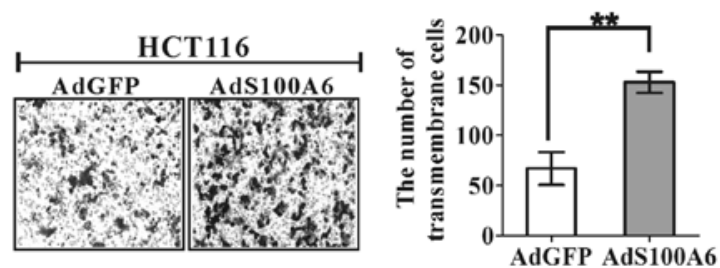

$\mathbf{E}$

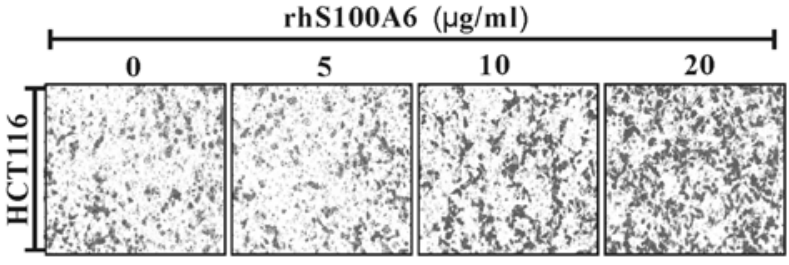

B
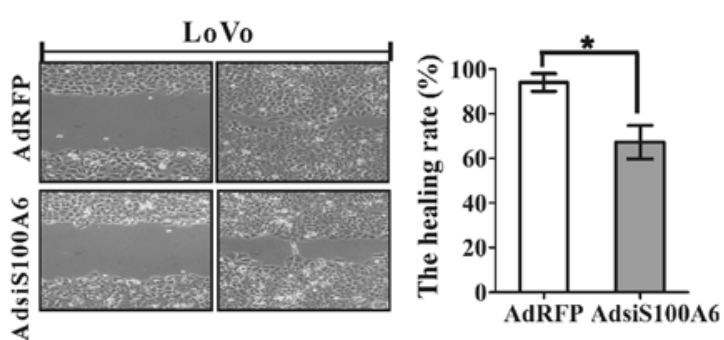

D
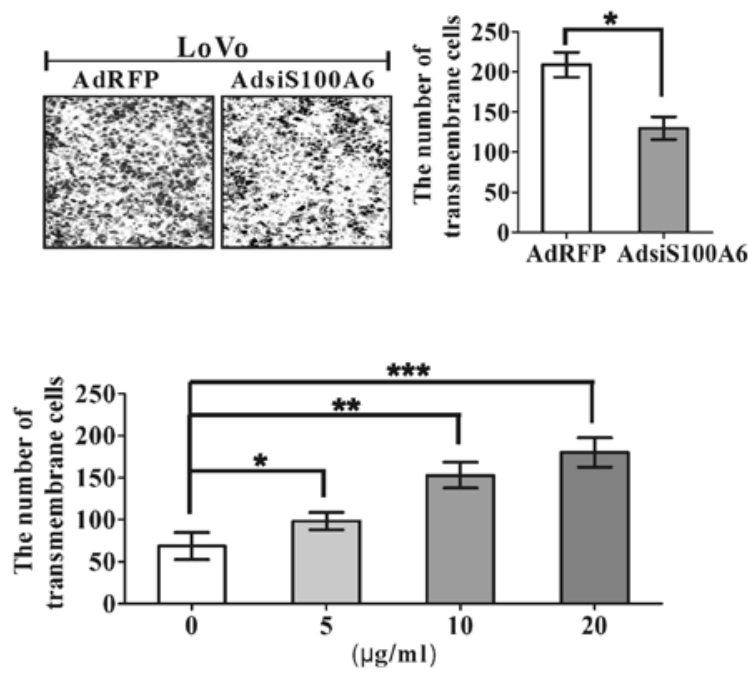

Figure 4. The effect of S100A6 on CRC cell migration. (A) Wound healing assay for analyzing the effect of AdS100A6 or AGFP on migration of HCT116 cells . The incision width of different sites was measured, and average healing rate was calculated. "p<0.05, AdS100A6 vs. AdGFP control. (B) Migration of LoVo cells of AdRFP- or AdsiS100A6-treated cells was detected by wound-healing assay. " $p<0.05$, AdsiS100A6 vs. AdRFP control. (C) Transwell migration assay for analyzing the migration of AGFP- or AdS100A6-treated HCT116 cells. Representative images of transmembrane cells are shown. The mean of transmembrane cells \pm SD per microscopic field of three independent experiments was calculated (right panel). Magnification, $x 100 .{ }^{* *}$ p $<0.01$, AdS100A6 vs. AdGFP control. (D) Transwell migration assay for analyzing the migration of ARFP- or AdsiS100A6-treated LoVo cells. Representative images of transmembrane cells are shown. The mean of transmembrane cells \pm SD per microscopic field of three independent experiments were quantified. Magnification, $x 100 .{ }^{*} p<0.05$, AdsiS100A6 vs. AdRFP control. (E) Transwell migration assay for analyzing the migration of HCT116 cells treated with rhS100A6 at different concentrations. Representative images of transmembrane cells are shown. The mean of transmembrane cells \pm SD per microscopic field of three independent experiments are quantified. Magnification, $\mathrm{x} 100 .{ }^{*} \mathrm{p}<0.05,{ }^{* *} \mathrm{p}<0.01$ and ${ }^{* * *} \mathrm{p}<0.001$, all vs. $\operatorname{rhS} 100 \mathrm{~A} 6(0 \mu \mathrm{g} / \mathrm{ml})$.

S100A6 with AdsiS100A6 in LoVo cells reduced wound closure rate at $72 \mathrm{~h}$ by $28.3 \%$ (p<0.05, Fig. 4B). The effects on cell migration by overexpression or knockdown of S100A6 were further confirmed by transwell migration assay, which showed that the number of transmembrane migrated HCT116 cells in the AdS100A6 group was increased by 1.28 -fold compared 
A
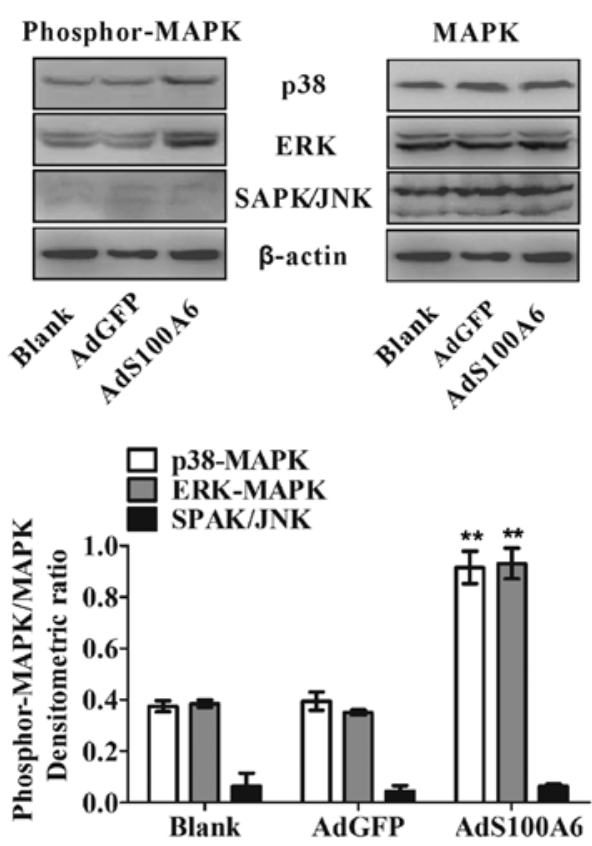

C Phosphor-MAPK

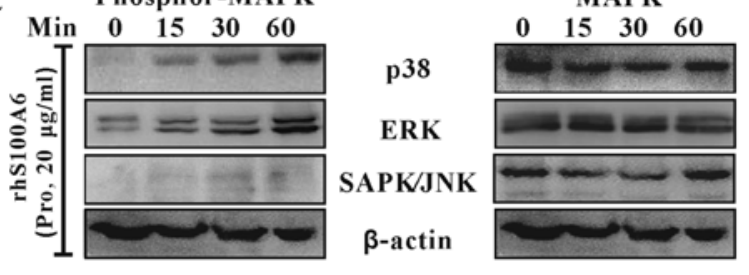

B
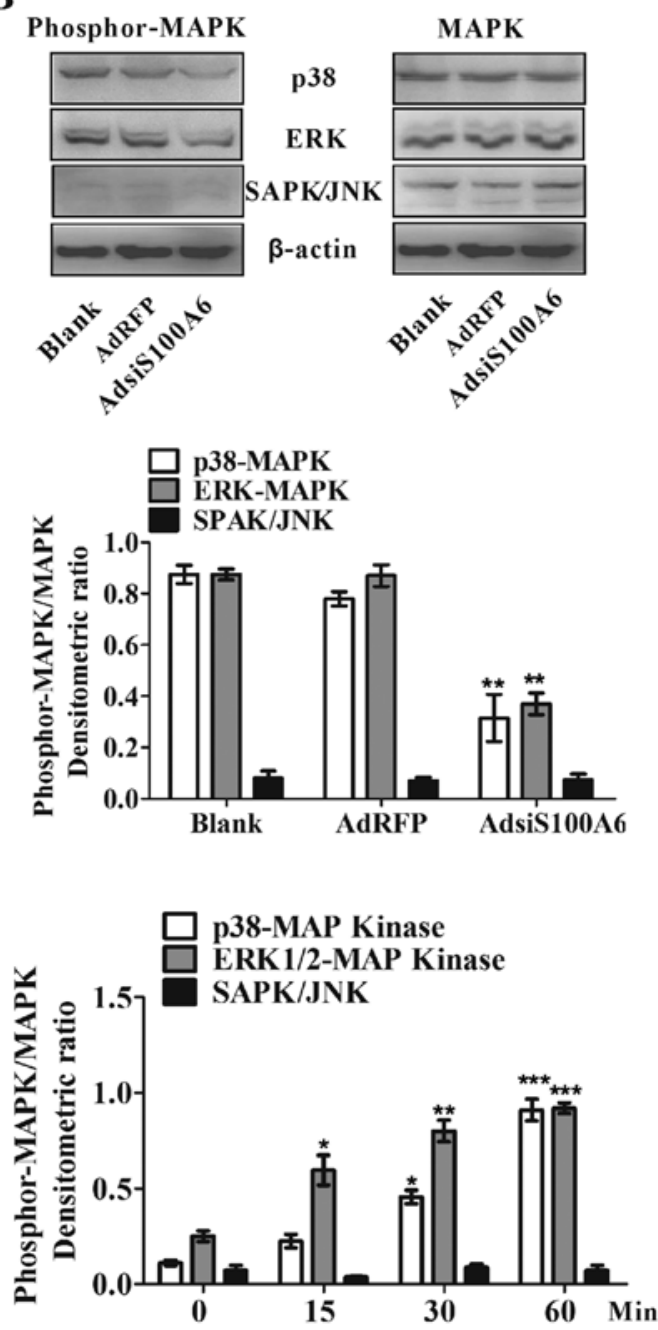

Figure 5. The effect of S100A6 on MAPK activation in CRC cells. (A) HCT116 cells were treated with or without AdS100A6 or AdGFP for 36 h. Phosphorylated MAPKs were detected by western blotting. Total p38, ERK1/2, SAPK/JNK and $\beta$-actin were included as loading controls. The densitometric ratios were normalized to the $\beta$-actin control. ** $\mathrm{p}<0.01$, AdS100A6 vs. AdGFP control. (B) Western blot analysis for phosphorylated MAPKs in LoVo cells treated with AdsiS100A6 or AdRFP for $36 \mathrm{~h}$. The densitometric ratios were normalized to the $\beta$-actin control. ${ }^{*}$ p $<0.01$, AdsiS100A6 vs. AdRFP control. (C) HCT116 cells were stimulated with rhS100A6 protein $(20 \mu \mathrm{g} / \mathrm{ml})$ for $0,15,30$ and $60 \mathrm{~min}$, and cell lysates were analyzed by western blot analysis using respective antibodies against phosphorylated MAPKs. Total p38, ERK1/2, SAPK/JNK and $\beta$-actin were included as the loading controls. The densitometric ratios were compared to the controls and then normalized to the $\beta$-actin control. Pro, protein. " $\mathrm{p}<0.05$, rhS100A6 (15 or $30 \mathrm{~min})$ vs. rhS100A6 (0 min); ${ }^{* *} \mathrm{p}<0.01$, rhS100A6 (30 min) vs. rhS100A6 (0 min) ${ }^{* * *} \mathrm{p}<0.001, \operatorname{rhS100A6}(60 \mathrm{~min})$ vs. $\operatorname{rhS100A6}(0 \mathrm{~min})$.

to that in the AdGFP group ( $\mathrm{p}<0.01$, Fig. $4 \mathrm{C})$, the number of transmembrane migrated LoVo cells was decreased by $38.1 \%$ compared to that in the AdRFP group ( $\mathrm{p}<0.05$, Fig. 4D). In addition, treatment of HCT116 cells with rhS100A6 protein also resulted in an increase in the migtation of HCT116 cells effectively in a concentration-dependent manner by transwell migration assay (Fig. 4E).

Activation of the MAPK signaling pathways in CRC cells by S100A6. Three mitogen-activated protein kinases (MAPKs): the extracellular-regulated kinase (ERK), p38 kinase, and the stress-activated protein kinase (SAPK)/c-jun N-terminal kinase (JNK) play a central role in cellular processes such as proliferation, differentiation, and tumorigenesis (28). To investigate whether S100A6 induces MAPK activation, we examined the phosphorylation of each MAPK in CRC cells with treatment of AdS100A6, rhS100A6 protein or AdsiS100A6 by western blotting. While overexpression of S100A6 by AdS100A6 in HCT116 cells had no detectable effect on phosphorylation of SAPK/JNK, it clearly enhanced the phosphorylation of p38 $(\mathrm{p}<0.01)$ and ERK1/2 (p<0.01) (Fig. 5A). Knockdown of S100A6 by AdsiS100A6 in LoVo cells decreased the phosphorylation of p38 $(\mathrm{p}<0.01)$ and ERK1/2 $(\mathrm{p}<0.01)$ while had no obvious effect on SAPK/JNK phosphorylation (Fig. 5B). In addition, treatment of HCT116 cells with rhS100A6 protein $(20 \mu \mathrm{g} / \mathrm{ml})$ also rapidly resulted in enhanced phosphorylation of p38 (within $30 \mathrm{~min}, \mathrm{p}<0.05$ ) and ERK1/2 (within $15 \mathrm{~min}$, $\mathrm{p}<0.05$ ) while it had no obvious effect on SAPK/JNK phosphorylation.(Fig. 5C).

Inhibition of ERK attenuates S100A6-induced proliferation and inhibition of 38 suppresses S100A6-induced migration. We next investigated the role of MAPK activation in S100A6induced CRC cell proliferation and migration. SB203580 and 
A
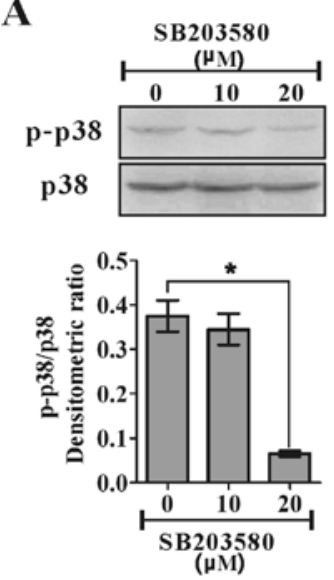

B
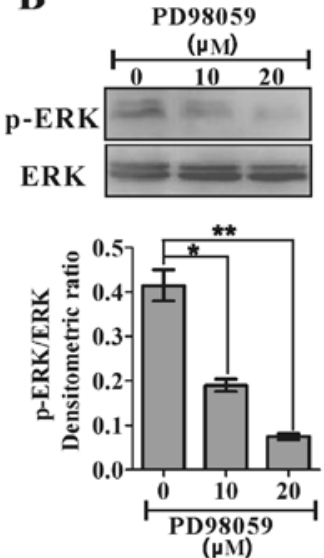

C

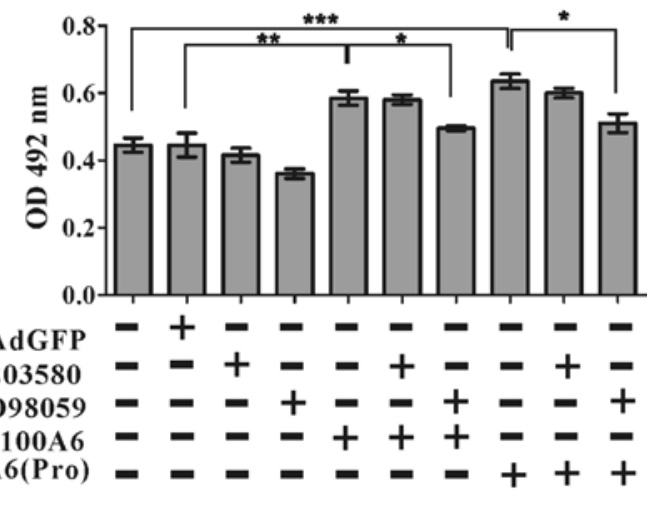

D

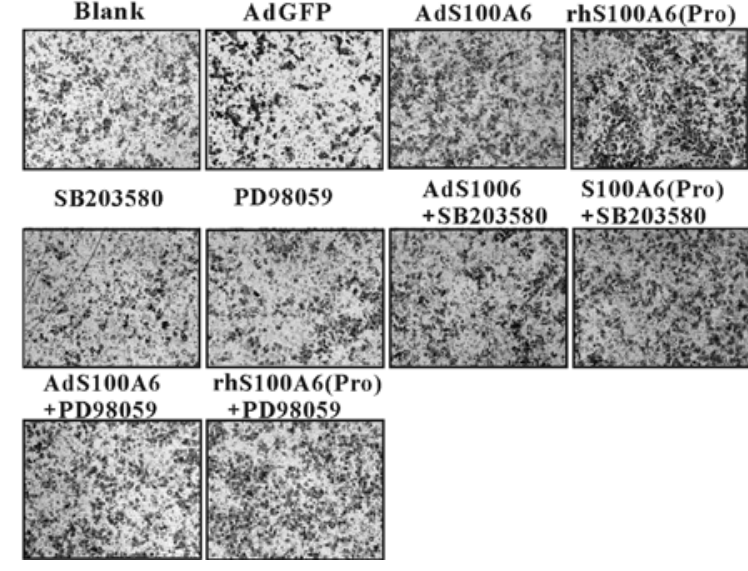

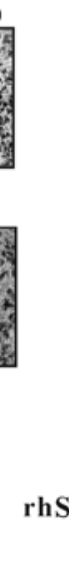
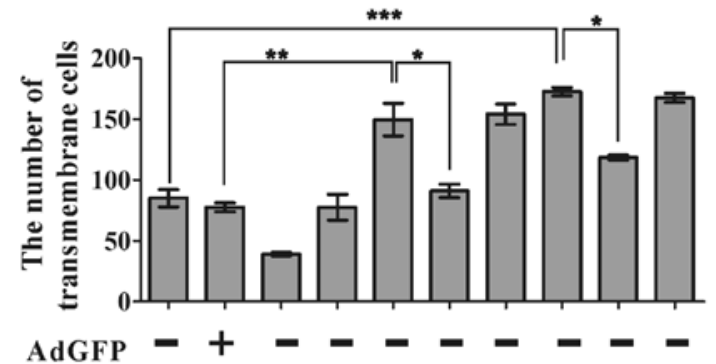

SB203580 $=+\bar{z}+\bar{z}+\bar{z}$

PD98059 $=-2+-2+-2+$

$\operatorname{AdS100A6}=--2+++-2$

Figure 6. Inhibition of ERK suppressed S100A6-indcued proliferation while inhibition of p38 MAPKs suppressed migration. (A) The HCT116 cells were treated with SB203580, and phosphorylation of p38 MAPK was detected by western blotting. The densitometric ratios were normalized to loading p38 MAPK control. "p $<0.01$, SB203580 $(0 \mu \mathrm{M})$ vs. SB203580 $(20 \mu \mathrm{M})$. (B) The cells were treated with PD98059 and phosphorylation of ERK was detected by western blotting. The densitometric ratios were normalized to loading total ERK control. " $\mathrm{p}<0.05$, PD98059 $(0 \mu \mathrm{M})$ vs. PD98059 (10 $\mu \mathrm{M})$; ${ }^{* *} \mathrm{p}<0.01$, PD98059 $(0 \mu \mathrm{M})$ vs. PD98059 $(20 \mu \mathrm{M})$. (C) The effect of SB203580 and PD98059 on S100A6-induced proliferation of HCT116 cells was detected by MTT assay. HCT116 cells were infected with AdGFP, AdS100A6 and rhS100A6 (20 $\mu \mathrm{g} / \mathrm{ml})$ followed by treatment with SB203580 (20 $\mu \mathrm{M})$ or PD98059 (20 $\mu \mathrm{M})$ for 4 days. Results are expressed as mean \pm SD of absorbance of three independent experiments at day 4. "p $<0.05$, AdS100A6 vs. AdS100A6+PD98059 or rhS100A6 vs. rhS100A6+PD98059; ${ }^{* *}$ p $<0.01$, AdS100A6 vs.AdGFP; ${ }^{* * *}$ p $<0.001$, rhS100A6 vs. blank (untreated). (D) The effect of SB203580 and PD98059 on S100A6-induced migration of HCT116 cells detected by transwell migration assay. HCT116 cells were infected with AdGFP, AdS100A6 and rhS100A6 (20 $\mu \mathrm{g} /$ $\mathrm{ml})$ followed by treatment with SB203580 $(20 \mu \mathrm{M})$ or PD98059 $(20 \mu \mathrm{M})$ for $24 \mathrm{~h}$. The representative images of transmembrane cells are shown (left panel), the mean \pm SD of migrated cell numbers per microscopic field of three independent experiments are quantified (right panel). Magnification, $\mathrm{x} 100 .{ }^{*} \mathrm{p}<0.05$, AdS100A6 vs. AdS100A6+SB203580 or rhS100A6 vs. rhS100A6+SB203580; ${ }^{* *}$ p <0.01, AdS100A6 vs.AdGFP; ${ }^{* * *}$ p <0.001, rhS100A6 vs. blank (untreated).

PD98059 were used, which effectively suppressed $\mathrm{p} 38(\mathrm{p}<0.05)$ and ERK1/2 (p<0.01), respectively (Fig. 6A). The enhanced proliferation by treatment with AdS100A6 or rhS100A6 protein $(20 \mu \mathrm{g} / \mathrm{ml})$ in HCT116 cells was partially reversed by the ERK inhibitor PD98059 $(\mathrm{p}<0.05)$ but not the p38 inhibitor SB203580 (Fig. 6B). In contrast, the enhanced migration of HCT116 cells induced by treatment with AdS100A6 or rhS100A6 protein $(20 \mu \mathrm{g} / \mathrm{ml})$ was partially reversed by SB203580 ( $<<0.05)$, but not PD98059 (Fig. 6C). These results suggest that the MAPKs have distinct roles in S100A6-induced CRC cell proliferation and migration.

\section{Discussion}

Several reports have demonstrated that S100A6 is involved in proliferation and motility of different tumor cells $(4,8,9)$. Although elevated expression of S100A6 in CRC was reported $(15,16)$, experimental evidence for functional involvement of
S100A6 in CRC tumor progression is still lacking. Here we provide data supporting a tumor-promoting role for S100A6 in CRC development, and that the ERK and p38 MAKs are involved in these functions of S100A6.

Increased S100A6 expression of in both CRC tissues and cell lines was confirmed by western blotting and immunohistostaining, which is consistent with previous studies demonstrating that increased S100A6 level was observed in numerous tumors such as squamous cell carcinoma of the mouth, pancreatic adenocarcinoma, gastric cancer and cutaneous epithelial tumors (29-32). Given its elevated expression in many tumors, S100A6 is likely involved in carcinogenesis.

We used the AdS100A6 and AdsiS100A6 to manipulate S100A6 expression in CRC cells, and used the rhS100A6 protein to manipulate its extracellular expression. Our results show that overexpression of S100A6 by AdS100A6 or treatment with rhS100A6 protein enhanced the proliferation of HCT116 cells, while knockdown of S100A6 by AdsiS100A6 in 
LoVo cells reduced cell proliferation, suggesting that S100A6 promotes proliferation of CRC cells. These results are in accordance with that from previous reports demonstrating that S100A6 contributes to proliferation of pulmonary fibroblast, osteoblasts and pancreatic carcinoma cells (5-7). We also found that S100A6 promotes CRC cell migration, which is consistent with previous reports showing S100A6 promotes pancreatic cancer cell motility, and knockdown of S100A6 resulted in a decrease of migration in fibroblast cells $(8,33)$. The cancer cell migration promotion property implies that S100A6 may be involved in CRC metastasis.

A growing body of evidence points to the importance of MAPK signaling in CRC progression. Activation of the p38 MAPK pathway participates in cell cycle arrest, autophagic cell death, and migration and invasion in CRC cells $(34,35)$. The ERK MAPK pathway mainly regulates cell proliferation in colorectal cancer while SPAK/JNK pathway is involved in regulation of apoptosis $(18,34,36)$. In the present study, we found that S100A6 stimulates the activation of p38 and ERK MAPKs in CRC cells. ERK mediated S100A6-induced proliferation while p38 mediated S100A6-induced the migration in CRC cells, respectively. These results are consistent with the reported functions of p38 and ERK MAPKs in regulating migration and proliferation of CRC cells.

Our data showed that treatment with AdS00A6 and rhS100A6 protein had similar roles in the proliferation, migration and activation of MAPK pathways in CRC cells. Based on this, previous studies showed S100A6 protein could be secreted from cells into the culture medium and interacted with RAGE thus exerting its promotive role in the survival of neuroblastoma cells $(4,11,37)$; moreover, RAGE expression and its downstream MAPK signaling have critical roles in intestinal tumorigenesis (11,38-42). Therefore we speculate that RAGE may mediate the enhanced proliferation, migration and activation of MAPK signaling by AdS100A6 or rhS100A6 protein in CRC. Confirmation requires future studies.

In conclusion, the current data indicate that S100A6 expression is elevated in CRC tissues and cell lines, and that S100A6 promotes the growth and migration of CRC cells by activating ERK1/2 and p38 MAPKs, respectively. Collectively, we provide important information regarding the role of S100A6 in the CRC progression, as a potential molecular target for developing cancer therapeutics.

\section{Acknowledgements}

The authors would like to thank Professor T.C. He (The University of Chicago, Medical Center) for his kind provision of the AdS100A6, AdGFP, AdsiS100A6 and AdRFP. The present study was supported by the National Natural Science Foundation of China (grant no. 30772548).

\section{References}

1. Siegel R, Naishadham D and Jemal A: Cancer statistics, 2013. CA Cancer J Clin 63: 11-30, 2013.

2. Meyerhardt JA, Li L, Sanoff HK, Carpenter W IV and Schrag D: Effectiveness of bevacizumab with first-line combination chemotherapy for Medicare patients with stage IV colorectal cancer. J Clin Oncol 30: 608-615, 2012.
3. Kuznicki J and Filipek A: Purification and properties of a novel $\mathrm{Ca}^{2+}$-binding protein $(10.5 \mathrm{kDa})$ from Ehrlich-ascites-tumour cells. Biochem J 247: 663-667, 1987.

4. Lesniak W, Slomnicki LP and Filipek A: S100A6 - new facts and features. Biochem Biophys Res Commun 390: 1087-1092, 2009.

5. Breen EC and Tang K: Calcyclin (S100A6) regulates pulmonary fibroblast proliferation, morphology, and cytoskeletal organization in vitro. J Cell Biochem 88: 848-854, 2003.

6. Hwang R, Lee EJ, Kim MH, et al: Calcyclin, a $\mathrm{Ca}^{2+}$ ion-binding protein, contributes to the anabolic effects of simvastatin on bone. J Biol Chem 279: 21239-21247, 2004.

7. Ohuchida K, Mizumoto K, Ishikawa N, et al: The role of S100A6 in pancreatic cancer development and its clinical implication as a diagnostic marker and therapeutic target. Clin Cancer Res 11: 7785-7793, 2005.

8. Nedjadi T, Kitteringham N, Campbell F, et al: S100A6 binds to annexin 2 in pancreatic cancer cells and promotes pancreatic cancer cell motility. Br J Cancer 101: 1145-1154, 2009.

9. Luo X, Sharff KA, Chen J, He TC and Luu HH: S100A6 expression and function in human osteosarcoma. Clin Orthop Relat Res 466: 2060-2070, 2008.

10. Mbeunkui F, Metge BJ, Shevde LA and Pannell LK: Identification of differentially secreted biomarkers using LC-MS/MS in isogenic cell lines representing a progression of breast cancer. J Proteome Res 6: 2993-3002, 2007.

11. Leclerc E, Fritz G, Weibel M, Heizmann CW and Galichet A: S100B and S100A6 differentially modulate cell survival by interacting with distinct RAGE (receptor for advanced glycation end products) immunoglobulin domains. J Biol Chem 282: 31317-31331, 2007.

12. Donato R: S100: a multigenic family of calcium-modulated proteins of the EF-hand type with intracellular and extracellular functional roles. Int J Biochem Cell Biol 33: 637-668, 2001.

13. Heizmann CW, Fritz G and Schafer BW: S100 proteins: structure, functions and pathology. Front Biosci 7: d1356-d1368, 2002.

14. Donato R: Intracellular and extracellular roles of S100 proteins. Microsc Res Tech 60: 540-551, 2003.

15. Komatsu K, Andoh A, Ishiguro S, et al: Increased expression of S100A6 (Calcyclin), a calcium-binding protein of the S100 family, in human colorectal adenocarcinomas. Clin Cancer Res 6: 172-177, 2000.

16. Alvarez-Chaver P, Rodriguez-Pineiro AM, RodriguezBerrocal FJ, Martinez-Zorzano VS and Paez de la Cadena M: Identification of hydrophobic proteins as biomarker candidates for colorectal cancer. Int J Biochem Cell Biol 39: 529-540, 2007.

17. Kilanczyk E, Graczyk A, Ostrowska H, Kasacka I, Lesniak W and Filipek A: S100A6 is transcriptionally regulated by betacatenin and interacts with a novel target, lamin $\mathrm{A} / \mathrm{C}$, in colorectal cancer cells. Cell Calcium 51: 470-477, 2012.

18. Fang JY and Richardson BC: The MAPK signalling pathways and colorectal cancer. Lancet Oncol 6: 322-327, 2005.

19. Tothova V and Gibadulinova A: S100P, a peculiar member of S100 family of calcium-binding proteins implicated in cancer. Acta Virol 57: 238-246, 2013.

20. Ghavami S, Rashedi I, Dattilo BM, et al: S100A8/A9 at low concentration promotes tumor cell growth via RAGE ligation and MAP kinase-dependent pathway. J Leukoc Biol 83: 1484-1492, 2008.

21. Kwon CH, Moon HJ, Park HJ, Choi JH and Park do Y: S100A8 and S100A9 promotes invasion and migration through p38 mitogen-activated protein kinase-dependent NF-kappaB activation in gastric cancer cells. Mol Cells 35: 226-234, 2013.

22. Wu R, Duan L, Ye L, et al: S100A9 promotes the proliferation and invasion of HepG2 hepatocellular carcinoma cells via the activation of the MAPK signaling pathway. Int J Oncol 42: 1001-1010, 2013.

23. Nakashima M, Sakai T, Hiraiwa H, et al: Role of S100A12 in the pathogenesis of osteoarthritis. Biochem Biophys Res Commun 422: 508-514, 2012.

24. Jin Q, Chen H, Luo A, Ding F and Liu Z: S100A14 stimulates cell proliferation and induces cell apoptosis at different concentrations via receptor for advanced glycation end products (RAGE). PloS One 6: e19375, 2011.

25. Duan L, Wu R, Ye L, et al: S100A8 and S100A9 are associated with colorectal carcinoma progression and contribute to colorectal carcinoma cell survival and migration via Wnt/betacatenin pathway. PloS One 8: e62092, 2013.

26. Zou Z, Wang H, Li Y, et al: Prokaryotic expression of recombinant protein HS100A6 and its biological effects on human osteosarcoma cell line 143B. China Biotechnol 32: 1-7, 2012. 
27. He TC, Zhou S, da Costa LT, Yu J, Kinzler KW and Vogelstein B: A simplified system for generating recombinant adenoviruses. Proc Natl Acad Sci USA 95: 2509-2514, 1998.

28. Seger R and Krebs EG: The MAPK signaling cascade. FASEB J 9: 726-735, 1995.

29. Berta GN, Ghezzo F, D'Avolio A, et al: Enhancement of calcyclin gene RNA expression in squamous cell carcinoma of the oral mucosa, but not in benign lesions. J Oral Pathol Med 26: 206-210, 1997.

30. Vimalachandran D, Greenhalf W, Thompson C, et al: High nuclear S100A6 (Calcyclin) is significantly associated with poor survival in pancreatic cancer patients. Cancer Res 65: 3218-3225, 2005.

31. Yang YQ, Zhang LJ, Dong H, et al: Upregulated expression of S100A6 in human gastric cancer. J Dig Dis 8: 186-193, 2007.

32. Fullen DR, Garrisi AJ, Sanders D and Thomas D: Expression of S100A6 protein in a broad spectrum of cutaneous tumors using tissue microarrays. J Cutan Pathol 35 (Suppl 2): 28-34, 2008.

33. Slomnicki LP and Lesniak W: S100A6 (calcyclin) deficiency induces senescence-like changes in cell cycle, morphology and functional characteristics of mouse NIH 3T3 fibroblasts. J Cell Biochem 109: 576-584, 2010.

34. Simone C: Signal-dependent control of autophagy and cell death in colorectal cancer cell: the role of the p38 pathway. Autophagy 3: 468-471, 2007.

35. Wei SC, Tsao PN, Weng MT, Cao Z and Wong JM: Flt-1 in colorectal cancer cells is required for the tumor invasive effect of placental growth factor through a p38-MMP9 pathway. J Biomed Sci 20: 39, 2013 .
36. Ragusa M, Statello L, Maugeri M, et al: Specific alterations of the microRNA transcriptome and global network structure in colorectal cancer after treatment with MAPK/ERK inhibitors. J Mol Med 90: 1421-1438, 2012.

37. Mohan SK, Gupta AA and Yu C: Interaction of the S100A6 mutant (C3S) with the V domain of the receptor for advanced glycation end products (RAGE). Biochem Biophys Res Commun 434: 328-333, 2013.

38. Heijmans J, Buller NV, Hoff E, et al: Rage signalling promotes intestinal tumourigenesis. Oncogene 32: 1202-1206, 2013.

39. Sasahira T, Akama Y, Fujii K and Kuniyasu H: Expression of receptor for advanced glycation end products and HMGB1/ amphoterin in colorectal adenomas. Virchows Arch 446: 411-415, 2005.

40. Kuniyasu H, Chihara Y and Takahashi T: Co-expression of receptor for advanced glycation end products and the ligand amphoterin associates closely with metastasis of colorectal cancer. Oncol Rep 10: 445-448, 2003.

41. Hofmann MA, Drury S, Fu C, et al: RAGE mediates a novel proinflammatory axis: a central cell surface receptor for $\mathrm{S} 100 /$ calgranulin polypeptides. Cell 97: 889-901, 1999.

42. Ichikawa M, Williams R, Wang L, Vogl T and Srikrishna G: S100A8/A9 activate key genes and pathways in colon tumor progression. Mol Cancer Res 9: 133-148, 2011. 